Neurosurg Focus 7 (5):Article 4, 1999

\title{
Microendoscopic posterior cervical foraminotomy: a cadaveric model and clinical application for cervical radiculopathy
}

\section{Timothy G. Burke, M.D., and Anthony Caputy, M.D.}

Department of Neurosugery, The George Washington University, Washington, D.C.

Cervical radiculopathy that is caused by either soft herniated disc material or foraminal stenosis is a common problem. Anterior and posterior surgical approaches are commonly performed to decompress the nerve root. The authors describe an endoscopic posterior foraminotomy procedure in which they use a rigid endoscope, in both a cadaveric model and in three clinical cases, including a multiple level case.

Postoperatively, all patients returned to functional work status within 4 weeks. The mean length of hospitalization was 1.3 days.

The advantages of this technique include improved visualization, a smaller incision, and significantly less postoperative discomfort when compared with a matched group of patients in whom open nonendoscopic foraminotomy has been performed.

Key Words * endoscopy * minimally invasive technique * posterior foraminotomy * radiculopathy * cervical spine

The treatment of cervical radiculopathy due to lateral compressive disease has traditionally been accomplished via an anterior or a posterior surgical approach.[2-4,6,7,9] There are several well-established series in which the efficacy of both techniques is demonstrated. Recently, major advances in endoscopic technology have stimulated efforts to treat spinal disorders by using the microendoscope. Although attention has primarily focused on the thoracic and lumbar spine, other groups have recently begun to initiate trials on cervical disc disease.[5] We describe a development in technique for performing endoscopic cervical foraminotomy in which a rigid glass endoscope is used. A significant improvement on the more commonly available fiberoptic system, this device provides superior visualization to any other modality in use today, including the surgical microscope.

This study will be used to establish the feasibility of performing microendoscopic posterior cervical foraminotomy in the clinical arena.

\section{CLINICAL MATERIALS AND METHODS}

\section{Cadaveric Study}


Three formalin-preserved and Micropaque-injected cadaver heads and cervical spines were used for this study. A two-level foraminotomy was performed in each sample (six on the right side and two on the left). The cervical level was not radiographically confirmed in the cadaver model.

A skin incision (10-14 $\mathrm{mm}$ in length) was made $5 \mathrm{~mm}$ off the midline in all specimens. The underlying tissue was sharply incised to the paraspinous muscles. These fibers were then spread in line by using a standard flat-blade nasal speculum. This was continued until the lamina-facet junction was reached. A self-retaining nasal speculum was then put in place for the rest of the procedure. The bony undersurface of the inferior border of the superior lamina was dissected using a small angled curette, and a laminotomy was performed, incorporating the adjacent lamina, using a 1- or 2-mm Kerrison rongeur. The superior and inferior pedicles were palpated using a small probe. A medial facetectomy was then performed using either a Kerrison punch or a special endoscopic suction/irrigation drill (Xomed, Jacksonville, FL). The drill possesses a cutting surface and a protective hood, which enables one to unroof the foramen while shielding the dura and nerve root from a cutting surface. Once an adequate decompression had been achieved, the nerve root was gently retracted and the disc space incised using a No. 11 blade scalpel. The disc material was removed using a small pituitary rongeur.

Click here to view QuickTime video clip.

Video Clip: A 2-mm Kerrrison ronguer is used to perform the initial laminotomy and partial facetectomy. A dissection around the nerve root is then conducted. The Xomed endoscopic drill is used to perform the foraminotomy. Note the appearance of the nerve root after the completion of the foraminotomy. Thereafter, a fragment of sequestered disc is dissected and removed with a small pituitary ronguer. Note the appearance of the nerve root after completion of decompression and removal of disc fragment.

\section{CLINICAL STUDY}

Three patients underwent microendoscopic posterior cervical foraminotomy. All patients provided informed consent, with full knowledge of all the potential risks and benefits of the procedure. All patients presented with radicular symptoms and signs (Table 1). Magnetic resonance imaging was performed in all patients. In one patient (Case 3) myelography and electromyelography were performed to confirm the affected nerve root.

\begin{tabular}{|c|c|c|c|c|}
\hline Sut & MMAR OF & $\begin{array}{l}\text { TABL } \\
\text { HARACTER STICS IN THREE P, } \\
\text { POSTERIOR CERYCA }\end{array}$ & $\begin{array}{l}\text { ENTS UND } \\
\text { ORAMINOT }\end{array}$ & $\begin{array}{l}\text { RGONG MICROENDOSCOPIC } \\
\text { MV }\end{array}$ \\
\hline $\begin{array}{l}\text { Case } \\
\text { No. }\end{array}$ & $\begin{array}{c}\text { Age }[(y+s) \\
\text { Sex }\end{array}$ & Symptorns & Duration & LevellDisc Fragments* \\
\hline $\begin{array}{l}1 \\
2 \\
3\end{array}$ & $\begin{array}{l}47, M \\
37, M \\
45, M\end{array}$ & $\begin{array}{l}\text { meakness, num bness, pain } \\
\text { pain, num tness } \\
\text { meakness, numbness, pain }\end{array}$ & $\begin{array}{l}1.5 \mathrm{yrs} \\
3 \mathrm{mos} \\
3 \mathrm{mos}\end{array}$ & $\begin{array}{l}\text { C6-7 babsent } \\
\text { C5-6iabsent; } 66-7 \text { /present } \\
\text { C6-7 babsent }\end{array}$ \\
\hline
\end{tabular}

In all patients surgery was performed in a semisitting position. A C-arm fluoroscopic device was positioned with the arc above the patient and left in place for the remainder of the procedure. A skin marker was placed to confirm the desired level based on the inferiorly located spinous process. A 10- to $15-\mathrm{mm}$ incision was marked in ink $5 \mathrm{~mm}$ off the midline, and this was followed by insertion of a spinal needle under fluoroscopic guidance. A trajectory to the facet joint was followed. A skin incision was 
made with a scalpel. Subcutaneous hemostasis and dissection were achieved with monopolar cautery, until the paraspinous musculature was reached. The fibers of the paraspinous muscles were split in the direction of their fibers by using a flat-blade nasal speculum. Once the bone of the lamina-facet border was reached, the rigid endoscope was brought into the field by the assistant (Storz 0 and 30, Karl Storz Endoscopy, Culver City, CA). At this point, the two adjacent laminae and facet joints were clearly seen, and the vertebral level was confirmed with fluoroscopy. A laminotomy was performed using a 1- or 2-mm Kerrison punch, and this was followed by identification of the superior and inferior pedicles (Fig. 1 upper left). The foraminal decompressive procedure was then performed (Fig. 1 upper right) using both Kerrison punches and the endoscopic drill (Xomed). Any bleeding originating from the epidural venous plexus was easily controlled with gelfoam (Pharmacea and Upjohn Co., Kalamazoo, MI), Avitene (Davol, Woburn, MA), and bipolar cautery. Once we were satisfied with the extent of the foraminal decompression, the nerve root was inspected and any adhesions were freed. We then gently retracted the nerve with a microdissector and searched for any sequestered disc material (Fig. 1 center left). If present, disc material was removed with a micropituitary rongeur (Fig. 1 center right). The nerve root was then inspected a final time to confirm that all compressive disc material had been removed. (Fig. 1 lower). Hemostasis was completed using an Avitene slurry. The incision was closed with two No. 2-0 vicryl sutures through the paraspinous fascia, two or three No. 3-0 vicryl sutures through the deep dermal layers, and skin closure was completed using a running No. 4-0 nylon or a subcuticular suture. 

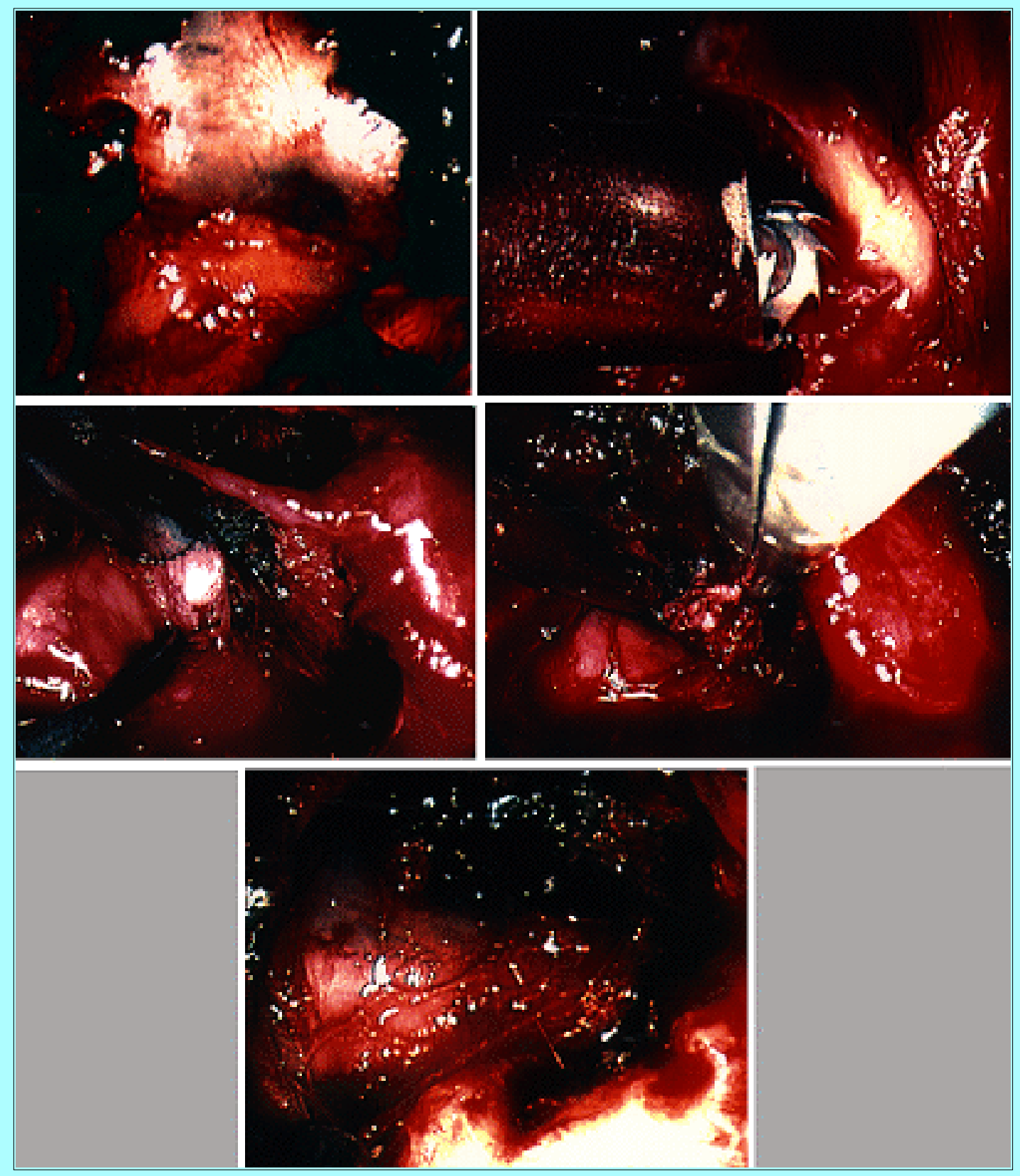

Fig. 1. Intraoperative photographs. Upper Left: A 2-mm Kerrison punch is used to perform the initial laminotomy and partial facetectomy. Upper Right: Further removal of the facet, along with foraminotomy is performed using the endoscopic drill. Center Left: Dissection of adhesion from an underlying fragment of disc material is performed. Center Right: A small pituitary rongeur is used to remove a fragment of sequestered disc material. Lower: The nerve root is shown after completion of foraminotomy and removal of a free fragment of disc material.

\section{RESULTS}

\section{Cadaveric Study}

In all specimens, the posterior elements were reached using the aforementioned techniques without difficulty. Removal of the bone was readily performed via the provided working channel by using standard Kerrison punches and the endoscopic drill. Bone decompression was visually confirmed at all operated levels. 


\section{Clinical Study}

Two of our three patients were found to have compression free fragments of disc material that caused compression. One patient (Case 2) underwent treatment of a herniated disc at an adjacent level (Table 1). The mean length of the procedure was 184 minutes (range 149-233 minutes). Blood loss in all three cases was minimal.

All patients tolerated the procedure well. Postoperatively they experienced improved radicular symptoms, with minimal neck discomfort. The mean hospital length of stay was 1.3 days. All patients returned to work within 1 month postoperatively, with one patient (Case 3) returning to work on postoperative Day 3.

Pathological findings in this small series confirmed free fragments of disc material in two of the first three patients. Disc fragments were removed without difficulty. One of these patients had an adjacent level of foraminal stenosis and underwent bone decompression only (Case 2). Patient 3 had only foraminal stenosis without significant disc herniation and underwent foraminotomy only.

\section{DISCUSSION}

It is well known that the benefit of the posterior approach to lateral disc herniations is that fusion is not required and that the risk of injurying anterior structures, such as the esophagus, carotid artery, and recurrent laryngeal nerve is avoided.[5,7,9] The major disadvantage to the posterior approach is that it is associated with significant postoperative neck discomfort.

Roh, et al., recently reported on a similar technique in a cadaver model using a fiberoptic system designed for the lumbar spine.[5] They were able to demonstrate that an equivalent amount of bone removal and exposure was possible with an endoscopic technique as compared with the standard keyhole method by performing computerized tomography scans of cadaver cervical spines following foraminotomies. The mean length of decompressed nerve root was $5.1 \mathrm{~mm}$ in the endoscopic technique verses $4.3 \mathrm{~mm}$ in the open laminotomy.[6] The major modification in this series is the use of the rigid endoscope. The improved optics of this system provides excellent visualization that more easily allows for substantial bone decompression.

The transition from a fiberoptic scope to the rigid endoscope is a major change that cannot be overemphasized. With the use of the latter system, we have now equaled and even surpassed the visualization quality of the surgical microscope. The first surgical assistant holds the endoscope. Several different types of endoscope holders have been used, but many of these are both cumbersome and expensive. The significantly smaller endoscope used in this series (4 mm diameter X $18 \mathrm{~cm}$ length) allows ample space for the use of two additional instruments in the operative field at any given time.The use of a nasal speculum or transphenoidal retractor has been found to be the most effective means of retraction. Commercially produced endoscopic spine-retraction systems lack the single functional channel that the speculum provides, which seems to give the most freedom of motion within a small area. In the initial cadaveric model, the facet was approached with a series of sequential dilators, ending with a singular tubular retractor. After several trials in which this method was used, the in-line fiber dissection and the nasal speculum were developed. Some degree of efficacy has been shown for the endoscopic drill, but the entire procedure can be performed using rongeurs without great difficulty.

One of the major concerns in the use of endoscopic techniques is that of managing bleeding.[5] In our three cases, we experienced no difficulty with visualizing and controlling sites of bleeding from the 
epidural venous plexus; we applied gelfoam with thrombin and Avitene slurry. It is not known whether control of bleeding might be more of an issue if the prone rather than semisitting position had been used.

The utility of this procedure was demonstrated in patients with adjacent levels of disease. A key point in performing two-level procedures is that the position of the incision must be made slightly to the inferior level to achieve the necessary angle for approaching the posterior elements. The size of the incision should be increased 2 to $3 \mathrm{~cm}$.

Several prospective and retrospective studies have been conducted on the subject of outcome after posterior cervical foraminotomy.[1,3-5,8] All of these series hold in common that the posterior approach to lateral disc lesions is an effective procedure in which a low morbidity rate was achieved in properly selected patients. In a review of 846 foraminotomies, Henderson and colleagues[3] found that the mean time to return to "normal activity" was 9.4 weeks. In our study of this small group, the patients were able to achieve this goal by 1 month postoperatively.

The results obtained in the three patients who underwent microendoscopic posterior foraminotomy were compared with those who underwent the traditional "keyhole" technique at the same institution. One of the concerns with any new procedure is the likelihood of lengthening the operative time. This concern is more acute in the foreground concerning endoscopic surgery. Our mean operative time was 184 minutes. The mean operative time for six prior cases in which open "keyhole" foraminotomy was performed was 236.5 minutes, a difference of $\mathbf{5 2 . 5}$ minutes. It is expected that as experience grows with this microendoscopic technique and technology evolves further that this operative time will continue to be reduced.

At a 3-month follow-up visit all patients had returned to work and to normal levels of activity including sporting activity. Radiculopathy had resolved in all, and strength has continued to improve in those who presented with motor deficits.

\section{CONCLUSIONS}

The microendoscopic posterior cervical foraminotomy is a viable, minimally invasive technique that provides exceptional visualization and an improvement in postoperative recovery time. As the quality of endoscopic equipment evolves and its availability increases, it is likely that this technique will ascend to a more dominant position in the rapidly changing field of minimally invasive surgery.

\section{References}

1. Davis RA: A long-term outcome study of 170 surgically treated patients with compressive cervical radiculopathy. Surg Neurol 46:523-533, 1996

2. Ebraheim NA, Xu R, Bhatti RA, et al: The projection of the cervical disc and uncinate process on the posterior aspect of the cervical spine. Surg Neurol 51:363-367, 1999

3. Henderson CM, Hennessy RG, Shuey HM Jr, et al: Posterior-lateral foraminotomy as an exclusive operative technique for cervical radiculopathy: a review of 846 consecutively operated cases.

Neurosurgery 13:504-512, 1983

4. Murphey F, Simmnos JCH, Brunson B: Surgical treatment of laterally ruptured cervical disc. J Neurosurg 38:679-683, 1973 
5. Roh SW, Kim DH, Cardoso AC, et al: Endoscopic foraminotomy using a microendoscopic discectomy system in cadaveric specimens. Neurosurg Focus 4 (2):Article 2, 1998

6. Scoville WB, Dohrmann GJ, Corkill G: Late results of cervical disc surgery. J Neurosurg 45:203-210, 1976

7. Williams RW: Microcervical foraminotomy. A surgical alternative for intractable radicular pain. Spine 8:708-716, 1983

8. Woertgen C, Holzschuh M, Rothoerl RD, et al: Prognostic factors of posterior cervical disc surgery: a prospective, consecutive study of 54 patients. Neurosurgery 40:724-729, 1997

9. Zdeblick TA, Zou D, Warden KE, et al: Cervical stability after foraminotomy. A biomechanical in vitro analysis. J Bone Joint Surg 74 (Am):22-27, 1992

Manuscript received September 17, 1999.

Accepted in final form October 27, 1999.

Address reprint requests to: Timothy G. Burke, M.D., Department of Neurosugery, The George Washington University, ACC 7-420, 2100 Pennsylvania Ave., NW, Washington, D.C. 20037. email: nevajcneu@gwumc.edu. 